\title{
Pedobacter suwonensis sp. nov., isolated from the rhizosphere of Chinese cabbage (Brassica campestris)
}

\author{
Soon-Wo Kwon, ${ }^{1}$ Byung-Yong Kim, ${ }^{1}$ Kang-Hyo Lee, ${ }^{2}$ Kab-Yeul Jang, ${ }^{2}$ \\ Soon-Ja Seok, ${ }^{2}$ Jang-Sik Kwon, ${ }^{2}$ Wan-Gyu Kim ${ }^{2}$ and Hang-Yeon Weon ${ }^{2}$

\begin{abstract}
${ }^{1}$ Korean Agricultural Culture Collection (KACC), Genetic Resources Division, National Institute of Agricultural Biotechnology, Rural Development Administration (RDA), Suwon 441-707, Korea

${ }^{2}$ Applied Microbiology Division, National Institute of Agricultural Science and Technology, RDA,
\end{abstract} \\ Suwon 441-707, Korea
}

Correspondence Hang-Yeon Weon hyweon@rda.go.kr

\begin{abstract}
A mesophilic bacterium, strain $15-52^{\top}$, was isolated from the rhizosphere of Chinese cabbage (Brassica campestris). On the basis of phenotypic and genotypic characteristics, the bacterium was identified as representing a novel species belonging to the genus Pedobacter. The strain is non-flagellated, non-spore-forming and grows at temperatures in the range $1-37^{\circ} \mathrm{C}$. Physiological tests of the strain showed the presence of oxidase, catalase, protease (gelatin and casein hydrolysis), $\beta$-glucosidase and $\beta$-galactosidase activities. The highest levels of $16 \mathrm{~S}$ rRNA gene sequence similarity were found with respect to Pedobacter roseus CL-GP80 ${ }^{\top}$ (97.3\%) and Pedobacter sandarakinus DS-27 $7^{\top}$ (97.2\%). A phylogenetic analysis based on $16 \mathrm{~S}$ rRNA gene sequence data indicated that strain $15-52^{\top}$ is a member of the genus Pedobacter. DNA-DNA hybridization analysis revealed low levels of relatedness $(<42.3 \%)$ between the isolate and two

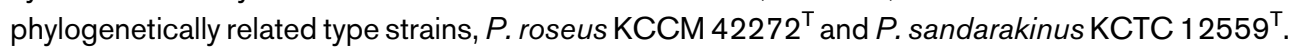
The DNA G $+\mathrm{C}$ content is $44.2 \mathrm{~mol} \%$ and the predominant fatty acids are iso- $\mathrm{C}_{15: 0}(35.4 \%)$, iso- $\mathrm{C}_{15: 0} 2-\mathrm{OH}$ and/or $\mathrm{C}_{16: 1} \omega 7 c(27.8 \%)$ and iso- $\mathrm{C}_{17: 0} 3-\mathrm{OH}(15.8 \%)$. On the basis of these data, strain $15-52^{\top}$ represents a novel species of the genus Pedobacter, for which the name Pedobacter suwonensis sp. nov. is proposed. The type strain is $15-52^{\top}\left(=\mathrm{KACC}_{11317^{\top}}=\mathrm{DSM}\right.$ $\left.18130^{\top}\right)$.
\end{abstract}

Pedobacter strains have been isolated from soils (Steyn et al., 1998; Yoon et al., 2006), water (Vanparys et al., 2005; Gallego et al., 2006; Hwang et al., 2006) and glaciers (Margesin et al., 2003; Shivaji et al., 2005). One distinctive characteristic of members of the genus is their ability to grow in temperatures in the range $1-37^{\circ} \mathrm{C}$. Their colonies exhibit various colours, from dirty yellow to creamy white or pink (Gallego et al., 2006), but the pigment does not produce the typical flexirubin reaction. Some species can degrade heparin: these include Pedobacter heparinus, Pedobacter africanus, Pedobacter saltans and Pedobacter himalayensis.

During the characterization of bacteria isolated from the rhizosphere of Chinese cabbage (Brassica campestris L.), we isolated a mesophilic strain, $15-52^{\mathrm{T}}$, belonging to the genus Pedobacter. Strain $15-52^{\mathrm{T}}$ was cultured on trypticase soy agar (TSA; Difco) at $30^{\circ} \mathrm{C}$ and maintained on TSA medium. The

The GenBank/EMBL/DDBJ accession number for the 16S rRNA gene sequence of strain $15-52^{\top}$ is DQ097274. cellular morphology was determined by using phasecontrast microscopy with 2 -day-old cells. Gliding motility was observed by direct microscopic examination of the edge of colonies grown on 1/10-strength trypticase soy broth (TSB) supplemented with $1 \%$ agar. Gram staining, catalase and oxidase activities, indole production and the hydrolysis of gelatin and aesculin were determined according to the methods of Smibert \& Krieg (1994). Heparinase production was determined by using the method of Joubert et al. (1984). Flexirubin pigments were tested for by suspending cells in $20 \% \mathrm{KOH}$ (Fautz \& Reichenbach, 1980). In addition, strain $15-52^{\mathrm{T}}$ was characterized by using the complete test spectrum provided by the API $20 \mathrm{NE}$ and API ZYM kits, according to the instructions of the manufacturer (bioMérieux). Carbon assimilation was tested by using the API $50 \mathrm{CH}$ gallery (bioMérieux), according to a standardized procedure (Kersters et al., 1984). The temperature ranges for the growth of strain $15-52^{\mathrm{T}}$ were determined by using a temperature-gradient incubator (TN-2612; Advantec) and TSB at $7-45^{\circ} \mathrm{C}$ (i.e. at $7,14,17,21,23,26$, $28,31,34,37,40$ and $\left.45^{\circ} \mathrm{C}\right)$; additional temperatures 
$\left(1-5^{\circ} \mathrm{C}\right)$ were tested with TSA slants by using a bench-top incubator (e-Cooling Bucket; Taitec).

Fatty acid methyl esters were extracted and prepared by using the standard protocol of the Microbial Identification System (MIDI; Microbial ID), with cells grown on TSA for $48 \mathrm{~h}$ at $28^{\circ} \mathrm{C}$. Isoprenoid quinones were analysed by HPLC as described previously (Groth et al., 1996). The DNA G + C content $(\mathrm{mol} \%)$ was determined by HPLC analysis of the deoxyribonucleosides, as described by Mesbah et al. (1989), using a reverse-phase column (Supelcosil LC-18-S; Supelco).

The 16S RNA gene sequence of strain $15-52^{\mathrm{T}}$ was analysed as described by Kwon et al. (2003). The sequences were aligned together with those of representative members of selected genera by using the CLUSTAL W program (Thompson et al., 1994). The evolutionary tree for the datasets was inferred from the neighbour-joining method of Saitou \& Nei (1987) by using MEGA version 3.1 (Kumar et al., 2004) (Fig. 1). The stability of relationships was assessed by performing bootstrap analyses of the neighbour-joining data, on the basis of 1000 resamplings. DNA-DNA hybridization was carried out using the filter hybridization method described by Seldin \& Dubnau (1985). Probe labelling was conducted by using the non-radioactive DIG-High Prime system (Roche), and hybridized DNA was visualized using the DIG luminescent detection kit (Roche). DNA-DNA relatedness was quantified by using a densitometer (Bio-Rad).

The phenotypic features that distinguish strain $15-52^{\mathrm{T}}$ from related Pedobacter species are shown in Table 1. The strain grew well on R2A, TSA and nutrient agar (Difco), but did not grow on MacConkey agar (Difco). The minimum doubling time of strain $15-52^{\mathrm{T}}$ at $34^{\circ} \mathrm{C}$ was about $2.6 \mathrm{~h}$. In API ZYM tests, strain $15-52^{\mathrm{T}}$ gave positive responses for 13 enzyme tests. In the API $50 \mathrm{CH}$ tests, the strain assimilated a total of 26 carbohydrates, including D-glycerol.

The predominant cellular fatty acids of strain $15-52^{\mathrm{T}}$ were iso- $\mathrm{C}_{15: 0}(35.4 \%)$, iso- $\mathrm{C}_{17: 0} 3-\mathrm{OH}(15.8 \%)$ and iso- $\mathrm{C}_{15: 0}$ $2-\mathrm{OH}$ and/or $\mathrm{C}_{16: 1} \omega 7 \mathrm{c}(27.8 \%$; the two fatty acids cannot be separated by GLC with the MIDI system) (Table 2).
Smaller amounts of iso- $\mathrm{C}_{17: 1} \omega 9 c(7.4 \%)$, iso- $\mathrm{C}_{15: 0} 3-\mathrm{OH}$ $(2.4 \%), \mathrm{C}_{16: 0}(1.6 \%), \mathrm{C}_{16: 1} \omega 5 c(1.4 \%)$ and $\mathrm{C}_{16: 0} 3-\mathrm{OH}$ $(1.2 \%)$ were also present. The DNA G $+\mathrm{C}$ content was $44.2 \mathrm{~mol} \%$, and the major respiratory quinones were MK-7 (90.5\%), MK-9 (5.6\%) and MK-8 (3.9\%).

Comparison of the 16S rRNA gene sequence of the strain with publicly available sequences of some Pedobacter strains showed that strain $15-52^{\mathrm{T}}$ clustered in a monophyletic group together with nine Pedobacter species (not including P. saltans) at a bootstrap value of $100 \%$. The strain showed the highest levels of 16S rRNA gene sequence similarity with respect to $P$. roseus CL-GP80 ${ }^{\mathrm{T}}(97.3 \%)$ and $P$. sandarakinus DS- $27^{\mathrm{T}}(97.2 \%)$. The levels of similarity between strain $15-52^{\mathrm{T}}$ and the type strains of other Pedobacter species ranged from $90.6 \%$ (P. saltans) to $95.9 \%$ (P. aquatilis). Strain $15-52^{\mathrm{T}}$ exhibited DNA-DNA reassociation values of 42.3 and $28.9 \%$, respectively, with respect to $P$. roseus KCCM $42272^{\mathrm{T}}$ and P. sandarakinus KCTC $12559^{\mathrm{T}}$, indicating that it is not related to them at the species level (Wayne et al., 1987).

On the basis of this polyphasic taxonomic approach, strain $15-52^{\mathrm{T}}$ represents a novel species of the genus Pedobacter, for which the name Pedobacter suwonensis sp. nov. is proposed.

\section{Description of Pedobacter suwonensis sp. nov.}

Pedobacter suwonensis (su.won.en'sis. N.L. masc. adj. suwonensis referring to Suwon, the city in which the type strain was isolated).

Cells are Gram-negative and rod-shaped $(0.65 \times 1.4-$ $3.2 \mu \mathrm{m})$. After 2 days on TSA, colonies are circular, 2-4 mm in diameter, smooth, convex with entire margins and pinkish yellow. Grows well on R2A, TSA and nutrient agar, but does not grow on MacConkey agar. Cells are aerobic, non-motile, non-spore-forming and mesophilic. Flexirubin-type pigments are absent. Growth occurs at pH 5.0-8.0 and $0-3 \% \mathrm{NaCl}$, with optimal growth at $\mathrm{pH} 6.5-7.0$ and $0-0.5 \% \mathrm{NaCl}$. Grows at temperatures in the range $1-37^{\circ} \mathrm{C}$, with optimum growth at $34^{\circ} \mathrm{C}$. Positive

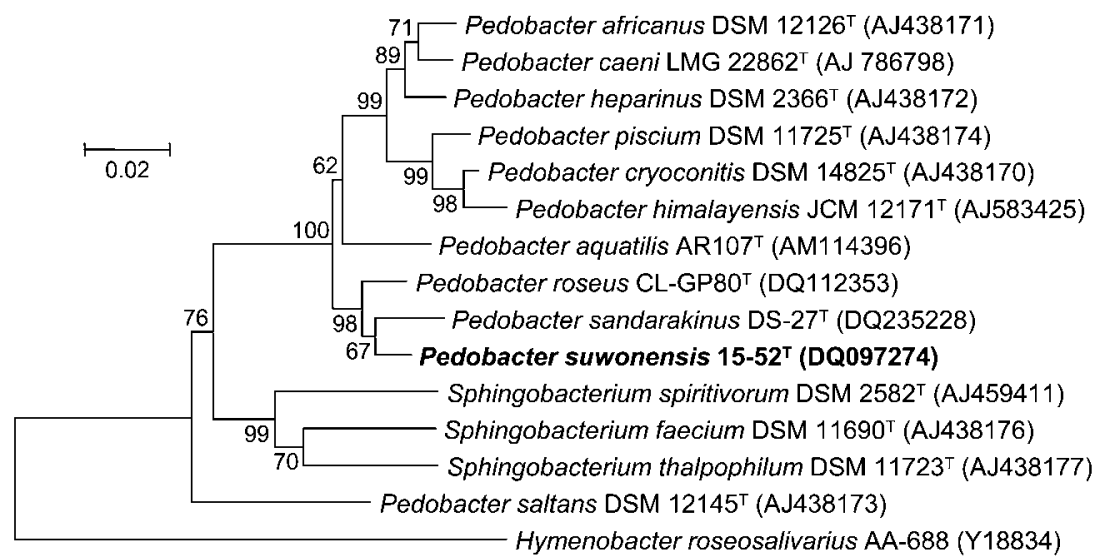

Fig. 1. Neighbour-joining phylogenetic tree, based on 16S rRNA gene sequences, showing the position of strain $15-52^{\top}$ with respect to Pedobacter species. Hymenobacter roseosalivarius AA-688 was used as the outgroup. Numbers at nodes indicate percentages of bootstrap support, based on 1000 resampled datasets; values below $50 \%$ are not shown. Bar, 2 substitutions per 100 nucleotides. 
Table 1. Phenotypic features that distinguish strain $15-52^{\top}$ from Pedobacter species

Taxa: 1, strain 15-52 ${ }^{\mathrm{T}}$; 2, P. himalayensis; 3, P. cryoconitis; 4, P. heparinus; 5, P. africanus; 6, P. piscium; 7, P. caeni; 8, P. sandarakinus; 9, P. aquatilis; 10, P. roseus. Data for reference species are from Steyn et al. (1998), Shivaji et al. (2005), Vanparys et al. (2005), Yoon et al. (2006), Gallego et al. (2006) and Hwang et al. (2006). +, Positive; -, negative; (+), weak; V, variable; ND, no data available. All taxa are positive for aerobic growth, the presence of catalase, oxidase, acid and alkaline phosphatases and $\beta$-galactosidase, aesculin hydrolysis and assimilation of D-cellobiose, D-glucose, D-mannose, lactose and $\mathrm{N}$-acetylglucosamine. All taxa are negative for the Gram stain and for sporulation, indole production, nitrate reduction, urease and assimilation of erythritol, L-sorbose, dulcitol and inositol.

\begin{tabular}{|c|c|c|c|c|c|c|c|c|c|c|}
\hline Characteristic & 1 & 2 & 3 & 4 & 5 & 6 & 7 & 8 & 9 & 10 \\
\hline Colony colour & $\begin{array}{l}\text { Pinkish } \\
\text { yellow }\end{array}$ & $\begin{array}{l}\text { Pale } \\
\text { white }\end{array}$ & $\begin{array}{l}\text { Creamy } \\
\text { white }\end{array}$ & $\begin{array}{c}\text { Translucent } \\
\text { yellow }\end{array}$ & $\begin{array}{c}\text { Translucent } \\
\text { yellow }\end{array}$ & $\begin{array}{c}\text { Yellow or } \\
\text { creamy white }\end{array}$ & Yellow & Orange & Pink & Pink \\
\hline \multicolumn{11}{|l|}{$\begin{array}{l}\text { Temperature for } \\
\text { growth }\left({ }^{\circ} \mathrm{C}\right)\end{array}$} \\
\hline Range & $1-37$ & $4-25$ & $1-25$ & $5-30$ & ND & $5-30$ & $?-37$ & $4-33$ & $4-30$ & $5-33$ \\
\hline Maximum & 37 & 25 & 25 & 37 & 37 & $25-30$ & 37 & 33 & 30 & 33 \\
\hline Motility & $\begin{array}{l}\text { Non- } \\
\text { motile }\end{array}$ & $\begin{array}{l}\text { Non- } \\
\text { motile }\end{array}$ & $\begin{array}{l}\text { Motile/ } \\
\text { gliding }\end{array}$ & Gliding & Non-motile & Gliding & Non-motile & Non-motile & Non-motile & Non-motile \\
\hline $\begin{array}{l}\text { Growth on } \\
\text { MacConkey agar }\end{array}$ & - & + & - & - & - & - & ND & - & - & + \\
\hline Gelatinase & + & + & + & - & $\mathrm{V}$ & - & - & - & - & + \\
\hline Heparinase & - & + & - & + & + & - & ND & ND & ND & - \\
\hline Lipase & - & - & - & - & + & + & - & - & - & - \\
\hline \multicolumn{11}{|l|}{$\begin{array}{l}\text { Assimilation of } \\
\text { carbon sources }\end{array}$} \\
\hline D-Glycerol & - & + & - & - & - & - & - & - & - & + \\
\hline D-Arabinose & - & + & - & - & $\mathrm{V}$ & - & - & - & - & ND \\
\hline L-Arabinose & + & + & + & + & V & + & + & - & + & + \\
\hline D-Ribose & - & + & - & - & $\mathrm{V}$ & - & - & - & - & - \\
\hline L-Xylose & - & + & - & - & - & - & + & - & - & + \\
\hline D-Adonitol & - & + & - & + & - & - & - & - & - & ND \\
\hline D-Galactose & + & + & + & + & + & $\mathrm{V}$ & - & + & + & + \\
\hline D-Fructose & $(+)$ & + & + & + & $\mathrm{V}$ & + & - & - & + & + \\
\hline L-Rhamnose & + & + & - & + & + & $\mathrm{V}$ & - & - & + & + \\
\hline D-Mannitol & - & + & - & + & - & - & - & - & - & - \\
\hline D-Sorbitol & - & + & - & + & - & - & - & - & - & - \\
\hline $\begin{array}{l}\text { Methyl } \\
\alpha \text {-D-mannoside }\end{array}$ & + & + & - & + & + & - & + & $(+)$ & + & $\mathrm{ND}$ \\
\hline Inulin & - & + & $(+)$ & - & - & - & - & - & - & + \\
\hline D-Melezitose & + & - & - & - & - & - & - & - & + & ND \\
\hline D-Raffinose & + & + & + & - & - & + & + & - & + & + \\
\hline Starch & + & + & + & - & $\mathrm{V}$ & + & + & + & + & ND \\
\hline Glycogen & + & - & + & - & - & - & - & + & - & + \\
\hline Xylitol & - & - & - & - & - & - & + & - & - & ND \\
\hline L-Fucose & - & + & - & + & V & - & - & - & - & ND \\
\hline $\begin{array}{l}\text { DNA G }+ \text { C } \\
\text { content }(\mathrm{mol} \%)\end{array}$ & 44.2 & 41.0 & 43.0 & $42-43$ & $43-45$ & $40-43$ & 42.7 & 39.7 & 38 & 41.3 \\
\hline
\end{tabular}

for catalase, oxidase, protease (hydrolysis of gelatin and casein) and aesculin hydrolysis. Negative for lipase, urease, arginine dihydrolase, indole production and nitrate reduction. Utilizes L-arabinose, D-xylose, D-galactose, D-glucose, D-fructose, D-mannose, L-rhamnose, methyl $\alpha$-D-mannoside, methyl $\alpha$-D-glucoside, $N$-acetylglucosamine, amygdalin, arbutin, aesculin, salicin, D-cellobiose, D-maltose, D-lactose, D-melibiose, sucrose, D-trehalose, D-melezitose,
D-raffinose, starch, glycogen, $\beta$-gentiobiose and D-turanose as sole carbon sources. Enzymic activity is observed for alkaline and acid phosphatase, esterase (C4), esterase lipase (C8), leucine arylamidase, valine arylamidase, trypsin, naphthol-AS-BI-phosphohydrolase, $\beta$-galactosidase, $\alpha$-glucosidase, $\beta$-glucosidase, $N$-acetyl- $\beta$-glucosaminidase and $\alpha$ fucosidase (API ZYM). The DNA G+C content is $44.2 \mathrm{~mol} \%$. The major menaquinone is MK-7 and the 
Table 2. Fatty acid compositions (\%) of strain $15-52^{\top}$ and related Pedobacter species

Taxa: 1, strain $15-52^{\mathrm{T}} ; 2$, P. himalayensis; 3, P. cryoconitis; 4, P. heparinus; 5, P. africanus; 6, P. piscium; 7, P. caeni; 8, P. sandarakinus; 9, P. aquatilis; 10, P. roseus. Data for reference strains are from Steyn et al. (1998), Shivaji et al. (2005), Vanparys et al. (2005), Yoon et al. (2006), Gallego et al. (2006) and Hwang et al. (2006). tr, Trace $(<1.0 \%)$; - , not detected/not reported.

\begin{tabular}{|c|c|c|c|c|c|c|c|c|c|c|}
\hline Fatty acid & 1 & 2 & 3 & 4 & 5 & 6 & 7 & 8 & 9 & 10 \\
\hline \multicolumn{11}{|l|}{$\begin{array}{l}\text { Straight-chain } \\
\text { saturated }\end{array}$} \\
\hline $\mathrm{C}_{14: 0}$ & $\operatorname{tr}$ & 1.0 & 1.0 & 1.1 & 1.5 & 1.3 & 2.5 & - & 1.0 & $\operatorname{tr}$ \\
\hline $\mathrm{C}_{15: 0}$ & - & - & - & 1.1 & $\operatorname{tr}$ & - & - & 4.2 & 1.6 & $\operatorname{tr}$ \\
\hline $\mathrm{C}_{16: 0}$ & 1.6 & 7.3 & 9.1 & 3.0 & 3.8 & 3.3 & 3.0 & - & 1.9 & $\operatorname{tr}$ \\
\hline $\mathrm{C}_{15: 0} 2-\mathrm{OH}$ & - & - & - & $\operatorname{tr}$ & $\operatorname{tr}$ & - & $\operatorname{tr}$ & 1.2 & - & $\operatorname{tr}$ \\
\hline $\mathrm{C}_{16: 0} 3-\mathrm{OH}$ & $\operatorname{tr}$ & - & - & 1.5 & 3.1 & 4.5 & 2.8 & - & 3.5 & 1.0 \\
\hline $\mathrm{C}_{17: 0} 2-\mathrm{OH}$ & - & - & - & 2.7 & $\operatorname{tr}$ & - & 1.3 & - & $\operatorname{tr}$ & $\operatorname{tr}$ \\
\hline \multicolumn{11}{|l|}{ Branched saturated } \\
\hline iso- $\mathrm{C}_{15: 0}$ & 35.4 & 33.0 & 15.0 & 28.2 & 26.6 & 26.2 & 21.3 & 35.8 & 28.9 & 37.0 \\
\hline iso- $\mathrm{C}_{17: 0}$ & - & - & - & - & - & - & - & 1.8 & - & - \\
\hline iso- $\mathrm{C}_{15: 0} 3-\mathrm{OH}$ & 2.4 & 1.5 & 1.9 & 2.5 & 2.1 & 2.5 & 3.3 & 3.0 & 2.7 & 2.7 \\
\hline iso- $\mathrm{C}_{17: 0} 3-\mathrm{OH}$ & 15.8 & 6.0 & 4.2 & 15.2 & 14.7 & 9.2 & 12.1 & 17.7 & 13.6 & 11.3 \\
\hline anteiso- $\mathrm{C}_{15: 0}$ & $\operatorname{tr}$ & - & - & $\operatorname{tr}$ & $\operatorname{tr}$ & 2.6 & $\operatorname{tr}$ & - & $\operatorname{tr}$ & 1.9 \\
\hline Summed feature $3^{*}$ & 27.2 & 22.0 & 29.0 & 30.6 & 34.1 & 42.1 & 39.9 & 21.7 & 30.7 & 24.5 \\
\hline \multicolumn{11}{|l|}{ Monounsaturated } \\
\hline $\mathrm{C}_{15: 1} \omega 6 c$ & - & - & - & - & $\operatorname{tr}$ & - & - & 1.6 & 2.1 & 1.6 \\
\hline $\mathrm{C}_{16: 1} \omega 5 c$ & 1.4 & - & - & 1.4 & 2.1 & 3.5 & 2.5 & - & 3.2 & 1.3 \\
\hline $\mathrm{C}_{17: 1} \omega 9 c$ & - & - & - & - & - & - & - & 1.1 & - & - \\
\hline iso- $\mathrm{C}_{17: 1} \omega 9 c$ & 7.4 & 4.0 & 8.6 & 6.3 & 4.4 & 1.6 & 2.2 & 3.8 & 2.6 & 7.6 \\
\hline anteiso- $\mathrm{C}_{17: 1} \omega 9 c$ & - & - & - & - & - & 1.2 & $\operatorname{tr}$ & - & - & - \\
\hline \multicolumn{11}{|l|}{ Unknown $\dagger$} \\
\hline ECL 13.56 & 1.3 & - & - & 5.0 & 2.6 & $\operatorname{tr}$ & - & - & - & 1.6 \\
\hline ECL 16.58 & 1.5 & - & - & 1.0 & 1.0 & $\operatorname{tr}$ & - & - & - & 1.5 \\
\hline
\end{tabular}

${ }^{*}$ Summed feature 3 contains iso- $\mathrm{C}_{15: 0} 2-\mathrm{OH}$ and/or $\mathrm{C}_{16: 1} \omega 7 c$ (the two fatty acids cannot be separated by GLC with the MIDI system).

$\dagger \mathrm{ECL}$, Equivalent chain length.

predominant cellular fatty acids are iso- $\mathrm{C}_{15: 0}$, iso- $\mathrm{C}_{17: 0}$ $3-\mathrm{OH}$ and iso- $\mathrm{C}_{15: 0} 2-\mathrm{OH}$ and/or $\mathrm{C}_{16: 1} \omega 7 c$.

The type strain, $15-52^{\mathrm{T}}\left(=\right.$ KACC $\left.11317^{\mathrm{T}}=\mathrm{DSM} 18130^{\mathrm{T}}\right)$, was isolated from the rhizosphere of Chinese cabbage (Brassica campestris L.).

\section{References}

Fautz, E. \& Reichenbach, H. (1980). A simple test for flexirubin-type pigments. FEMS Microbiol Lett 8, 87-91.

Gallego, V., Garcia, M. T. \& Ventosa, A. (2006). Pedobacter aquatilis sp. nov., a novel bacterium isolated from drinking water, and emended description of the genus Pedobacter. Int J Syst Evol Microbiol 56, 1853-1858.

Groth, I., Schumann, P., Weiss, N., Martin, K. \& Rainey, F. A. (1996). Agrococcus jenensis gen. nov., sp. nov., a new genus of actinomycetes with diaminobutyric acid in the cell wall. Int J Syst Bacteriol 46, 234-239.
Hwang, C. Y., Choi, D. H. \& Cho, B. C. (2006). Pedobacter roseus sp. nov., isolated from a hypertrophic pond. Int J Syst Evol Microbiol 56, 1831-1836.

Joubert, J. J., van Rensburg, E. J. \& Pitout, M. J. (1984). A plate method for demonstrating the breakdown of heparin and chondroitin sulphate by bacteria. J Microbiol Methods 2, 197-202.

Kersters, K., Hinz, K.-H., Hertle, A., Segers, P., Lievens, A., Siegmann, O. \& De Ley, J. (1984). Bordetella avium sp. nov., isolated from the respiratory tracts of turkeys and other birds. Int J Syst Bacteriol 34, 56-70.

Kumar, S., Tamura, K. \& Nei, M. (2004). MEGA3: integrated software for molecular evolutionary genetics analysis and sequence alignment. Brief Bioinform 5, 150-163.

Kwon, S. W., Kim, J. S., Park, I. C., Yoon, S. H., Park, D. H., Lim, C. K. \& Go, S. J. (2003). Pseudomonas koreensis sp. nov., Pseudomonas umsongensis sp. nov. and Pseudomonas jinjuensis sp. nov., novel species from farm soils in Korea. Int J Syst Evol Microbiol 53, 21-27.

Margesin, R., Spröer, C., Schumann, P. \& Schinner, F. (2003). Pedobacter cryoconitis sp. nov., a facultative psychrophile from alpine glacier cryoconite. Int J Syst Evol Microbiol 53, 1291-1296. 
Mesbah, M., Premachandran, U. \& Whitman, W. B. (1989). Precise measurement of the $\mathrm{G}+\mathrm{C}$ content of deoxyribonucleic acid by highperformance liquid chromatography. Int J Syst Bacteriol 39, 159-167.

Saitou, N. \& Nei, M. (1987). The neighbor-joining method: a new method for reconstructing phylogenetic trees. Mol Biol Evol 4, 406-425.

Seldin, L. \& Dubnau, D. (1985). Deoxyribonucleic acid homology among Bacillus polymyxa, Bacillus macerans, Bacillus azotofixans, and other nitrogen-fixing Bacillus strains. Int J Syst Bacteriol 35, 151-154.

Shivaji, S., Chaturvedi, P., Reddy, G. S. N. \& Suresh, K. (2005). Pedobacter himalayensis sp. nov., from the Hamta glacier located in the Himalayan mountain ranges of India. Int J Syst Evol Microbiol 55, 1083-1088.

Smibert, R. M. \& Krieg, N. R. (1994). Phenotypic characterization. In Methods for General and Molecular Bacteriology, pp. 607-654. Edited by P. Gerhardt, R. G. E. Murray, W. A. Wood \& N. R. Krieg. Washington, DC: American Society for Microbiology.

Steyn, P. L., Segers, P., Vancanneyt, M., Sandra, P., Kersters, K. \& Joubert, J. J. (1998). Classification of heparinolytic bacteria into a new genus, Pedobacter, comprising four species: Pedobacter heparinus comb. nov., Pedobacter piscium comb. nov., Pedobacter africanus sp. nov. and Pedobacter saltans sp. nov. Proposal of the family Sphingobacteriaceae fam. nov. Int J Syst Bacteriol 48, 165-177.

Thompson, J. D., Higgins, D. G. \& Gibson, T. J. (1994). CLUSTAL W: improving the sensitivity of progressive multiple sequence alignment through sequence weighting, position-specific gap penalties and weight matrix choice. Nucleic Acids Res 22, 4673-4680.

Vanparys, B., Heylen, K., Lebbe, L. \& De Vos, P. (2005). Pedobacter caeni sp. nov., a novel species isolated from a nitrifying inoculum. Int J Syst Evol Microbiol 55, 1315-1318.

Wayne, L. G., Brenner, D. J., Colwell, R. R., Grimont, P. A. D., Kandler, O., Krichevsky, M. I., Moore, L. H., Moore, W. E. C., Murray, R. G. E. \& other authors (1987). International Committee on Systematic Bacteriology. Report of the ad hoc committee on reconciliation of approaches to bacterial systematics. Int $J$ Syst Bacteriol 37, 463-464.

Yoon, J.-H., Lee, M.-H., Kang, S.-J., Park, S.-Y. \& Oh, T.-K. (2006). Pedobacter sandarakinus sp. nov., isolated from soil. Int J Syst Evol Microbiol 56, 1273-1277. 